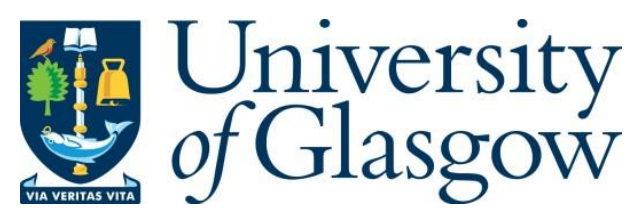

Krämer, S. (2017) Everything, and then some. Mind, 126(502), pp. 499-528.

There may be differences between this version and the published version. You are advised to consult the publisher's version if you wish to cite from it.

http://eprints.gla.ac.uk/140559/

Deposited on: 4 May 2017

Enlighten - Research publications by members of the University of Glasgow http://eprints.gla.ac.uk 


\title{
Everything, and then some
}

Higher-Orderism and the Spirit and Letter of Generality Absolutism

\author{
[author] ${ }^{*}$
}

This article has been accepted for publication by Mind, published by Oxford University Press.

Please consult the published version for citations.

Abstract: On its intended interpretation, logical, mathematical, and metaphysical discourse sometimes seems to involve absolutely unrestricted quantification. Yet our standard semantic theories do not allow for interpretations of a language as expressing absolute generality. A prominent strategy for defending absolute generality, influentially proposed by Timothy Williamson in his paper 'Everything' (2003), avails itself of a hierarchy of quantifiers of ever increasing orders to develop non-standard semantic theories that do provide for such interpretations. However, as emphasized by Øystein Linnebo and Agustín Rayo (2012), there is pressure on this view to extend the quantificational hierarchy beyond the finite levels, and, relatedly, to allow for a cumulative conception of the hierarchy. In his recent book Modal Logic as Metaphysics (2013), Williamson yields to that pressure. I show that the emerging cumulative higher-orderist theory has implications of a strongly generality-relativist flavour and consequently undermines much of the spirit of generality absolutism that Williamson set out to defend.

[Acknowledgements.] 


\section{Introduction}

In ordinary discourse, most of our quantifications are restricted to a set of contextually relevant objects. I say 'there is no beer', meaning not that there is absolutely no beer in the entire universe, but that there is no beer in my fridge, and thus no contextually relevant beer. In logical, metaphysical, and mathematical discourse, in contrast, we often seem to generalize without any such restrictions. In typical utterances of 'nothing has contradictory properties', 'everything is self-identical', 'everything is either abstract or concrete', or 'nothing is a member of the empty set', it seems, absolutely nothing is excluded as contextually irrelevant.

However, the appearance that absolute generality can thus be expressed comes under pressure from a number of theoretical considerations. ${ }^{1}$ The one that is most important for our purposes is that in our most successful and best understood semantic theories, quantification is always interpreted with respect some set that constitutes the presumed domain of discourse. Since there is no universal set, these semantic theories do not allow for absolute generality. Some philosophers hold that this apparent limitation of such theories cannot be overcome in a satisfactory manner, and have therefore embraced (Generality) Relativism, the view that initial appearances notwithstanding, there can be no such thing as absolutely general discourse. ${ }^{2}$

In his paper 'Everything' (2003), Timothy Williamson mounts a forceful defence of the opposition to Relativism, i.e. (Generality) Absolutism. As part of this defence, he proposes an alternative kind of semantics in which object language quantifiers need not be interpreted as restricted to a set. ${ }^{3}$ The crucial move that enables him to avoid this limitation is the employment of higher-order quantification in the meta-language. ${ }^{4}$

1 For an excellent overview of the debate, including a discussion of other arguments against the possibility of absolute generality, see the editors' introduction to Rayo and Uzquiano, 2006 as well as Florio, 2014.

2 Sometimes in the debate over absolute generality a distinction is made between a metaphysical question - roughly, whether there is an all-inclusive domain of discourse - and a linguistic or availability question - roughly, whether we could quantify over such a domain, if it exists (cp. e.g. Rayo and Uzquiano, 2006, 2, who credit Kit Fine with having first emphasized this point). The distinction, if it can be made, is not of particular importance for our present purposes. As I understand the distinction, our discussion concerns the availability question throughout.

3 It may be objected that familiar truth-theoretic semantics formulated in the Davidson-Tarski style have no need to interpret object language quantifiers as restricted to a set. That is of course correct; the difficulty arises when we try to formulate a theory that specifies the truth-conditions of the object-language sentences for arbitrary interpretations of the language. We shall come back to this point shortly.

4 The same kind of move was already employed for a similar objective by George Boolos (1985) and, 
As Williamson notes, the obvious generalization of his proposal requires the use of at least the whole finite hierarchy of orders of quantification. Moreover, as Øystein Linnebo and Agustín Rayo (2012) have shown, given a number of plausible further assumptions, we need to countenance even quantification of transfinite and cumulative orders. And when Williamson returns to the issue in his Modal Logic as Metaphysics (2013), he explicitly avails himself of such quantifiers.

The purpose of this paper is to show that the move to transfinite, cumulative orders of quantification undermines much of the spirit, if not the letter, of the Absolutist picture that Williamson wishes to defend. Moreover, since the relevant components of Williamson's view are difficult or impossible to reject given his basic approach of using higher-order resources to defend absolute generality, the argument also presents a severe challenge for any view following this general approach. I first present the current state of the debate. $\$ 2$ describes the problem that Absolutists face when trying to formulate an adequate semantics for absolutely general quantification. $\$ 3$ presents Williamson's higher-orderist solution to the problem and then explains both the notion of quantification at transfinite and cumulative orders and why Williamson avails himself of such quantifiers. $\mathbb{S} 4$ develops my argument that the cumulative higher-order picture has implications strongly reminiscent of Relativism. I first state the argument in informal terms and indicate the obstacles to formalizing it in the non-cumulative higher-orderist's canonical language. I then show how the introduction of cumulative resources allow us to overcome these obstacles. The crucial bit of cumulative ideology that we need is a higher-order, cumulative analogue of the notion of identity. That notion is discussed in more detail in $\$ 5$. Finally, I ask to what extent the problems Williamson raises for Relativism have counterparts that apply to his own cumulative higher-orderist version of Absolutism. $\$ 6$ points out that cumulative higher-orderism faces a similar difficulty as standard Relativism concerning the adequate formulation of its apparent Relativist commitments. $\$ 7$ turns to the criticism of standard Relativism that its restricted interpretations often seem to constitute weird misinterpretations of relevant object-language discourse. I show that this criticism also applies on the cumulative higher-orderist picture, though in a somewhat less dramatic form. I conclude in $\$ 8$ that Williamson's cumulative higher-orderist Absolutism is a lot closer to Relativism than it first appears.

further developing Boolos's suggestions, by in Rayo and Uzquiano, 1999. 


\section{The semantic challenge for absolutism}

A peculiar feature of the dispute over absolute generality is that it is not very easy to say just what it takes to be an Absolutist, or a Relativist, who is worthy of the name. ${ }^{5}$ Take Absolutism. It is not enough to say: 'It is possible to quantify over absolutely everything', or even:

(1) I am now quantifying over absolutely everything.

The reason is that everyone can say that, since any utterance of (1) will express a truth. For whatever the quantifier phrase 'absolutely everything' ranges over in such an utterance, what the speaker then claims is merely that they are quantifying over everything in that range, which cannot fail to be true. However, if the quantifier phrase ranges over only a restricted domain, the claim made by the speaker, though true, is irrelevant to the spirit, if perhaps not the letter, of Absolutism. It is only if the quantifier phrase ranges over absolutely everything that the speaker makes a claim that is relevant to the debate, and such that accepting it makes one an Absolutist proper.

If our speaker is to count as an Absolutist, therefore, we shall require them to back up their utterance of (1) by insisting, in a meta-linguistic utterance, on an interpretation of their utterance on which it generalizes over everything. And while our speaker remained firmly on the safe side with their utterance of (1), once they back it up in this way in the meta-language, as Williamson shows, they face a real threat of contradiction. First, let us shift our focus slightly by considering formal languages instead of utterances of English sentences like (1). Presumably, if it can be consistently maintained of an utterance of (1) that it generalizes over everything, then it is possible to specify a formal language of which it can be consistently maintained that its quantifiers range over everything. We shall therefore assume that the Absolutist commits to the following claim:

(GA) It is possible to specify a formal language containing quantifiers that, from the point of view of a suitable meta-language, range over absolutely everything.

A language $L^{\prime}$ is a suitable meta-language for a formal language $L$ if it is possible to develop an adequate semantic theory for $L$ in $L^{\prime}$.

5 On this issue, cf. Williamson, 2003, $\$$ V, Florio, 2014, 2, Rayo and Uzquiano, 2006, 2f, and the references given there. 
A second assumption we shall make has to do with the kind of semantic theorizing about the object language that we want to be possible in our meta-language. We shall assume that for any formal language, it is possible to construct what we may call a generalized semantics ${ }^{6}$ for that language. A generalized semantics for a language is a semantic theory which provides an inductive characterization of the truth-conditions of the object-language sentences, relative to arbitrary ways of interpreting (at least) their non-logical constants. (A generalized semantics is therefore exactly the kind of theory we require as the basis for a broadly model-theoretic theory of logical consequence.) Our second assumption can thus be described as a principle of Semantic Optimism:

(SO) For any formal language $L$, it is in principle possible to construct a theory adequately specifying the truth-conditions of sentences of $L$ relative to arbitrary ways of interpreting $L$ 's non-logical constants.

This principle is by no means trivial. Still, in the debate about absolute generality, it is standardly assumed that the principle at least enjoys a high degree of initial plausibility (cf. e.g. Linnebo, 2006, 150, Linnebo and Rayo, 2012, 276f). For the purposes of this paper, I shall therefore take it for granted. The challenge for the Absolutist is then to specify a formal language, and to formulate a generalized semantics for that language, so that from the point of view of the meta-language, the quantifiers of the object-language range over absolutely everything. Unfortunately, there is a powerful argument purporting to show that this cannot be done. ${ }^{7}$

Suppose that the Absolutist has described a formal language $L_{1}$ whose (first-order) quantifiers they wish to maintain express absolute generality. The Absolutist must now formulate a generalized semantics for $L_{1}$ in a suitable meta-language. A generalized semantics is supposed to specify the truth-conditions of the sentences of $L_{1}$ relative to arbitrary ways of interpreting (the non-logical constants of) $L_{1}$. It therefore needs to generalize over (things that model) ways of interpreting $L_{1}$. For simplicity, assume that ' $i$ ' is a meta-language variable that ranges over whatever the semantics uses to model ways of interpreting $L_{1}$.

Now take some monadic predicate $P$ of $L_{1}$. It seems very plausible that we can in principle use any contentful monadic predicate of the meta-language to interpret $P$. If

6 The way I here set up the dispute is indebted to Linnebo 2006 and Linnebo and Rayo 2012, from where I have also borrowed my terminology.

7 Except for minor details, the argument to follow is Williamson's (2003, 425ff). 
so, then any instance of the following Comprehension schema for Predicate Interpretations obtained by replacing ' $F$ ' with such a meta-language predicate should be true.

(CPI Informal) Under at least one way of interpreting the non-logical constants of $L_{1}$, $P$ applies to all and only the $F s$ in the range of $L_{1}$ 's quantifiers.

The semantics should then imply a suitable regimentation of (CPI Informal) in the meta-language. For brevity, we add copies ' $\forall$ ' ' and ' $\exists$ ' ' of $L_{1}$ 's first-order quantifiers to the meta-language. We may then formulate a suitable regimentation of (CPI Informal) as follows, using 'applies ${ }_{i}$ ' to abbreviate 'applies under interpretation $i$ ':

(CPI Formal 1) $\exists i \forall_{o} x\left(P\right.$ applies $_{i}$ to $\left.x \leftrightarrow F x\right)$

The Absolutist also has to include in their theory an expression of the claim that from the point of the semantics, $L_{1}$ 's quantifiers range over absolutely everything. An obvious way to formalize that claim is:

$\left(\mathrm{GA}_{1}\right) \forall y \exists_{o} x x=y$

However, these assumptions jointly entail a contradiction. For consider the following instance of (CPI Formal 1):

(2) $\exists i \forall_{o} x\left(P\right.$ applies $_{i}$ to $x$ iff $\neg P$ applies $_{x}$ to $\left.x\right)$

Assuming that some interpretation $i^{\prime}$ verifies (2), we have:

(3) $\forall_{o} x\left(P\right.$ applies $_{i}$ to $x$ iff $\neg P$ applies $_{x}$ to $\left.x\right)$

By $\left(\mathrm{GA}_{1}\right)$, the range of ' $\forall$ ' ' comprises everything. So in particular, it includes $i$ '. We may therefore instantiate (3) with $i^{\prime}$ to obtain:

(4) $P$ applies $_{i^{\prime}}$ to $i^{\prime}$ iff $\neg P$ applies $_{i^{\prime}}$ to $i^{\prime}$

But (4) is equivalent in classical logic to an explicit contradiction.

The Relativist maintains that it is the Absolutist's claim $\left(\mathrm{GA}_{1}\right)$ which is to blame for the contradiction, and concludes that there is more than is dreamt of by $L_{1}$ 's quantifiers: $\left(\mathrm{MORE}_{1}\right) \exists y \forall_{0} x x \neq y$

To be an Absolutist, in contrast, one must keep $\left(\mathrm{GA}_{1}\right)$, and thus find some other way out. The next section explains how Williamson proposes to do that.

$8 \quad$ The variable ' $i$ ' is not allowed to occur free in a substituend for ' $F$ '. 


\section{Higher-orderist absolutism}

Our above regimentation of (CPI Informal) by (CPI Formal 1) embodies a tacit assumption to the effect that the informal talk of ways of interpreting an object language predicate is to be understood in first-order quantificational terms. After all, (CPI Formal 1) uses a first-order quantifier ' $\exists i$ ' presumed to range over objects of some sort that are identified with, or taken to represent, ways of interpreting object language predicates. Williamson suggests that this tacit assumption is mistaken; it is more plausible, according to him, to represent ways of interpreting predicates by means of a secondorder variable (cf. 2003, 452ff). In effect, his proposal has us replace (CPI Formal 1) with a second-order analogue, in which the first-order variable ' $i$ ' and the quantifier binding it have been replaced by a second-order variable and quantifier: ${ }^{9}$

(CPI Formal 2) $\exists I \forall_{o} x\left(P\right.$ applies $_{I}$ to $\left.x \leftrightarrow F x\right)$

The semantic predicate 'applies,' may be thought of as defined by:

(Df. applies $_{I}$ ) $P$ applies to $x \leftrightarrow I(P, x)$

Note that from the standard (full) axiom scheme of comprehension for second-order logic, we obtain every instance of the schema

(5) $\exists I \forall_{o} x(I(P, x) \longleftrightarrow F x)$

in which the expression replacing ' $F$ ' does not contain ' $I$ ' free. This in turn guarantees the validity of (CPI Formal 2).

At least at first glance, it seems to me, this approach to the semantic challenge for Absolutism - call it the higher-orderist approach - has a lot to be said for it. What the Russell-paradoxical argument from (2) to (4) shows, we might say, is that there are always strictly more ways to interpret a predicate with respect to a domain of objects than there are objects in that domain, and thus ways to interpret a first-order variable with respect to that domain. ${ }^{10}$ As a result, not every way to interpret a predicate with respect to a given domain can be represented by an object in that domain. The Relativist concludes from this that there must always be objects outside any domain

9 Similarly as for (CPI Formal 1), instantiation of ' $F$ ' with a predicate in which ' $I$ ' ' is free is not allowed.

10 The talk of domains is not to be taken too literally. There is no need to assume that the objects that a predicate is interpreted as true or false of are members of some further object that we call a domain. 
with respect to which a predicate can be interpreted. The higher-orderist approach, in contrast, concludes that it is a mistake to try to represent ways of interpreting a predicate by the values of first-order variables. Instead, the thought goes, we should use second-order variables for that purpose. For crucially, there is no obstacle to holding that there are always at most as many ways to interpret a predicate with respect to a domain of objects as there are ways to interpret a predicate variable with respect to that domain.

It is important to note, however, that the proposal depends on a very specific view - call it higher-orderism - of second- and higher-order quantification. Before stating the view, let me clarify my talk of quantifiers of first, second, and higher orders. For present purposes, that classification is to be thought of in syntactic terms. On this understanding, what makes the familiar quantifiers ' $\exists x$ ' and ' $\forall x$ ' of $L_{1}$ first-order is that they bind variables that stand in the syntactic position of singular terms. What we shall call second-order quantifiers are then quantifiers that bind variables standing in the syntactic position of expressions that form sentences when combined with one or more singular terms as their arguments, i.e. ordinary predicates. A third-order quantifier, by analogy, is a quantifier binding variables that take the position of expressions forming sentences when combined with ordinary predicates as their arguments. The hierarchy extends in the obvious way to quantifiers and variables of order $n$ for any finite $n$.

Higher-orderism can now be defined as the conjunction of the following three theses concerning this syntactic hierarchy: (1) Quantification of any finite order is a legitimate linguistic device. (2) Quantifications of a given order are not in general paraphrasable by quantifications of a lower order. (3) For any finite $n$, adequate semantic clauses for $n$ 'thorder quantifications themselves employ $n$ 'th-order quantifiers of the meta-language in the way standard clauses for first-order quantifiers employ first-order quantifiers of the meta-language. ${ }^{11}$

To get an idea why the Williamsonian proposal requires the full strength of higherorderism, note first that if second-order quantification were in general paraphrasable by first-order quantification, then in particular (CPI Formal 2) could be paraphrased in first-order terms. But such a paraphrase would reintroduce the inconsistency engendered by (CPI Formal 1). Moreover, given (SO), we can develop a generalized semantics

11 I am not claiming that the three theses are independent, but it is a non-trivial question what entailment relations may obtain between them, and so it seems best to stay neutral with respect to that question in characterizing higher-orderism. 
for our second-order meta-language. If we were to do that in a first-order meta-language, perhaps by construing second-order quantifiers as ranging over properties, we should again run into a version of Russell's Paradox. Indeed, by a higher-order analogue of the above Russell-style argument, it can be shown that a generalized semantics for a second-order language cannot be given even in a second-order language: we need to use third-order resources. ${ }^{12}$ So Williamson's higher-orderist approach depends on the legitimacy and irreducibility of quantification of every finite order.

The irreducibilism embodied in higher-orderism has important implications for how we can read second- and higher-order quantifications in natural language. For example, at first glance the second-order quantification ' $\exists X \forall x \neg X x$ ' might naturally be read as 'some property is had by no object'. However, although often useful and appropriate for heuristic purposes, such a reading cannot be considered strictly adequate on the higher-orderist view. The reason is that the English quantifier here used to interpret the formal higher-order quantifier is itself first-order. This can be seen from the fact that if we ask for a witnessing instance of the quantification, grammar demands that the answer consist in a singular noun phrase like 'the property of being self-distinct' rather than a predicate.

It is controversial whether one can translate second-order quantification into natural language in a way that fits higher-orderism. ${ }^{13}$ For our purposes, it does not matter too much, for even if this is possible, it seems clear that natural languages do not provide us with the resources needed to appropriately translate quantifiers of arbitrarily high finite orders. So higher-orderists must hold that we can, at least in principle, somehow come to understand third-, fourth-, and higher-order quantification without the benefit of a translation into vocabulary that we independently understand. ${ }^{14}$ In what follows, to avoid excessive formalism and for heuristic purposes, we shall make use of various natural language constructions to approximate the higher-orderists' intended interpretation of their vocabulary, bearing in mind, though, that these may occasionally yield a

12 Strictly speaking, the situation is slightly more complicated; it is examined in detail in Rayo, 2006. The essential point remains, however. Semantic optimism forces the higher-orderist up the hierarchy of meta-languages, and if they are to permit the formulation of a generalized semantics in accordance with the higher-orderist approach, the meta-languages must include quantificational devices of ever increasing finite orders.

13 George Boolos famously proposed a translation using English plural quantification (1984, 1985); an alternative, more predicational reading was first suggested by Arthur Prior (1971, ch. 3) and recently developed in more detail by Rayo and Yablo, 2001.

14

Compare Williamson, 2003, 457ff, Linnebo, 2006, $152 \mathrm{ff}$. 
slightly misleading picture of the higher-orderist view. We shall grant, moreover, both the truth of higher-orderism, and the claim that higher-orderism provides sufficient expressive resources to formulate a generalized semantics for any language of finite order, i.e. such that for some finite $n$, it contains no quantifiers of an order higher than $n$.

So far, I have merely described the explicit key commitments of the higher-orderist defence of absolute generality as described for example in Williamson 2003 and Rayo 2006. We now turn to two crucial further claims that Williamson endorses, implicitly at least, in his recent book (2013). The first claim is that in addition to the languages of finite order that we have already canvassed, there is also a legitimate language that contains quantifiers of every finite order. With respect to the ordering of languages of higher and higher finite orders, this language would occupy the level of the first limit ordinal $\omega$, so we may call it $L_{\omega}$. Williamson uses a language like this as his favoured background language for metaphysical theorizing and so is obviously committed to considering such a language legitimate. ${ }^{15}$

The question whether the higher-orderist defence of absolute generality on its own is committed to this claim is more difficult to answer. Certainly, the legitimacy of $L_{\omega}$ does not follow logically from anything the higher-orderist has said so far. Nevertheless, it would seem prima facie quite implausible to disallow it. ${ }^{16}$ For every bit of vocabulary we find in $L_{\omega}$ has already been deemed legitimate, since it is also found in some 'successor' language in the higher-orderist's hierarchy. And it is hard to see how pooling all these individually coherent and legitimate linguistic resources together into a single language could somehow fail to produce an equally coherent and legitimate language. The burden of proof, therefore, would seem to lie with anyone wishing to deny the legitimacy of the limit language $L_{\omega}$.

If a $L_{\omega}$ is allowed, then by (SO), it is possible to give a generalized semantics for it. The second crucial claim of Williamson's is that such a semantics can be stated using quantification of transfinite orders. Note first that higher-orderism, as defined above, does not by itself provide sufficient resources to formulate a semantics for $L_{\omega}$ in accordance with the higher-orderist approach. For on this approach, to interpret expressions of order $n$, we need to make use of an interpretation variable of at least order $n$. But every kind of variable higher-orderism provides us with belongs to some

15 The modal higher-order language $\mathrm{ML}_{P}$ that Williamson proposes in 2013, ch. V contains quantifiers of every finite order and is thus relevantly like $L_{\omega}$.

16 On this point, see also Linnebo and Rayo, 2012, $275 f$ and Rayo, 2006, $246 f f$. 
finite order $n$. And for every finite order $n$, since $L_{\omega}$ contains quantifiers and variables of every finite order, it contains variables of order $n+1$. So no interpretation variable of a finite order can be used to interpret all the expressions of $L_{\omega}$. What Williamson does, therefore, in specifying a semantics for a limit language, is avail himself of predicates, and quantifiable predicate variables, of transfinite orders. ${ }^{17}$

Aside from their transfinite character, Williamson's predicates and predicate variables of transfinite order have a further striking feature that sets them apart from any of the expressions we have encountered so far. That feature concerns what expressions they accept as arguments. Usually, a predicate or predicate variable accepts as arguments expressions of the next lower order: a first-order predicate accepts singular terms, a second-order predicate accepts first-order predicates, and so on. This rule cannot extend to our new predicates, though. For these are of order $\omega$, and there is no next lower order to $\omega$. Instead, these predicates accept expressions of any lower, i.e. finite order in their argument place. I shall therefore describe these new predicates as syntactically cumulative. It turns out that a syntactically cumulative $\omega$-order predicate variable is just what is needed, and just what Williamson uses, for an interpretation variable in a generalized semantics for $L_{\omega}{ }^{18}$

Let me stress that for the purposes of this paper, the admission of cumulative resources is the important point. The admission of $L_{\omega}$ and transfinite orders of quantification matter only in virtue of their bearing on this point. And while the above consideration provides perhaps the most principled and compelling case for the legitimacy of cumulativity, it should be noted that even independently of limit languages and transfinite orders of quantification, higher-orderists may have reason to be sympathetic to this claim. For since they allow quantification of every finite order, they are already committed to a fairly liberal standard for admissible linguistic devices. And

17 See Williamson, 2013, 236ff. I do not know whether the move to transfinite orders is strictly the only way to give a generalized semantics for a language like $L_{\omega}$, as seems to be suggested by the discussion in Linnebo and Rayo, 2012, 275, and appendix B. For all I know, it might also be possible to use separate interpretation variables for every order, which could then themselves all be of finite order. However, we would then have to relativize satisfation to infinitely many parameters, so we should have to introduce predicates with infinitely many argument places, as well as quantifiers binding an infinite set of variables. I do not know how a semantics would have to look like that is adequate for this kind of infinitary language. I cannot rule out entirely, however, that there might be a coherent version of higher-orderism that allows $L_{\omega}$ but no transfinite orders of quantification. Thanks here to [blinded] and [blinded].

18 The construction is given in Williamson, 2013, 236ff. A more detailed presentation and discussion of similar constructions is given in the appendices ofLinnebo and Rayo 2012. 
from a logico-mathematical point of view, cumulative resources make perfect sense and have a principled and well-behaved logic. ${ }^{19}$ So it is not at all clear that there is a defensible standard of intelligibility that could serve to rule out cumulative devices, without at the same time ruling out quantifiers of very high finite orders.

\section{Cumulative higher-orderism and the spirit of absolutism}

Let us return from the dizzy heights of $\omega$-order quantification to the first and simplest stage in the higher-orderist's hierarchy of languages and semantic theories: the generalized semantics for the first-order language $L_{1}$, formulated in a second-order metalanguage. Recall the sentence that started us off on the whole higher-order journey, our formalization of the claim that $L_{1}$ 's quantifiers are, from the point of view of the semantics for $L_{1}$, absolutely general:

$\left(\mathrm{GA}_{1}\right) \forall y \exists_{0} x x=y$

This section develops an argument that according to cumulative higher-orderism, even though the higher-order semantics for $L_{1}$ includes $\left(\mathrm{GA}_{1}\right)$, it does not make the quantifiers of $L_{1}$ absolutely general. More precisely, I argue that for a cumulative higherorderist, there is a good sense in which

(MORE) From the point of view of the higher-order semantics for $L_{1}$, there is more than is quantified over in $L_{1}$.

I shall begin by sketching the argument in informal terms.

In the setting of the original, first-order semantics for $L_{1},\left(\mathrm{GA}_{1}\right)$ constitutes an adequate formalization of the claim that $L_{1}$ 's quantifiers are absolutely general. For the quantifier ' $\forall y$ ' ranges over absolutely every bit of reality that is countenanced in that semantics, and so $\left(\mathrm{GA}_{1}\right)$ says of absolutely everything countenanced in the semantics that it is in the reach of the quantifiers of $L_{1}$. However, that situation changes when the higher-orderist proceeds to extend the language of the semantics by second-order quantifiers. Since the higher-orderist insists that these are in no way reducible to first-order ones, we have to see them as concerned with new bits of reality that are not in the range

19 On this point, see also Linnebo and Rayo, 2012, 278. For an in-depth discussion of the logicomathematical properties of cumulative higher-order logic, see Degen and Johannsen 2000. 
of the semantics' first-order quantifiers. But then ' $\forall y$ ' no longer ranges over absolutely every bit of reality that is countenanced in that semantics. Consequently, $\left(\mathrm{GA}_{1}\right)$ is no longer a plausible formalization of the absolute generality of $L_{1}$ 's quantifiers. Even on the higher-orderist picture, there is more than is dreamt of by $L_{1}$ 's quantifiers - what sets the picture apart from the Relativist's is only that what's more is not in the range of the semantics' first-order quantifiers but their second-order cousins. So the higherorderist version of Absolutism is not really worthy of the name. In subscribing to $\left(\mathrm{GA}_{1}\right)$, it preserves the letter of Absolutism, but in implying (MORE), it gives up on its spirit.

Cast as it is in informal terms, the objection so far inspires limited confidence. After all, the higher-orderist has warned us that informal, natural language approximations of their higher-order quantifications can be misleading. Can we put the informal objection on a more rigorous footing by reproducing it in a formal setting congenial to higherorderism? More specifically, can we find a plausible formalization of (MORE) which is a consequence of the higher-orderist semantics?

Here is a somewhat flat-footed argument that we cannot: To say, as the objection alleges, that there is more, on the higher-orderist view, than is included in the range of $L_{1}$ 's quantifiers, we should have to say that there is something which is distinct from everything in that range. The condition of being distinct from everything in that range is expressed by

$$
\left(\neq_{1}\right) \forall_{0} x \ldots x
$$

Now to say that there is something satisfying that condition, one has to put a variable into the gap of $\left(\neq_{1}\right)$, and bind it by an existential quantifier. If the result is to be wellformed, however, we can only put a first-order variable into the gap of $\left(\neq_{1}\right)$. But the putative extra bits of reality countenanced by the higher-orderist are supposed to be introduced only by second-order quantifiers. Such a quantifier, however, cannot bind the first-order variable in the gap of $\left(f_{1}\right)$. So there is no sense in which, according to higher-orderism, there is something more than is ranged over by the quantifiers in $L_{1}$. Any attempt to even formulate that claim in the higher-orderist's canonical language produces an ill-formed string. In that language, as Williamson puts it, 'quantification into predicate position is simply incommensurable with quantification into name position; the former presents no coherent threat to the absolute generality of the latter' (Williamson, 2003, 458). 
As I have stated it, this line of reasoning depends on the following assumption:

(DIST) A regimentation of (MORE) contradicts the spirit of Absolutism only if it uses $\left(\neq_{1}\right)$ to express distinctness from anything in the range of the object-language quantifier.

I shall argue that this assumption is implausible, and that once we are allowed to make use of cumulative higher-order resources, second-order quantification ceases to be incommensurable with first-order quantification, and does present a serious threat to the latter's absolute generality. In a first step, I show that (DIST) should be rejected even independently of any issues to do with cumulativity. In a second step, I present a number of relatively modest ways to extend the higher-orderist's second-order meta-language with cumulative vocabulary, and show that in this extended cumulative higher-orderist setting, we can formulate and prove a well-formed regimentation of (MORE) that clashes with the spirit Absolutism.

It will help if I first set up a system of grammatical types that allows us to describe the syntax of cumulative as well as non-cumulative expressions. For ease of comparison of the resources I employ to those used by Williamson, I base my system on the one he uses (cf. 2013, 221). It has just one basic type $e$, which is the type of singular terms. Then whenever $t_{1}, \ldots, t_{n}$ are types, $\left\langle t_{1}, \ldots, t_{n}\right\rangle$ is the derived (functional) type of expressions that form sentences when combined with $n$ further expressions of types $t_{1}, \ldots, t_{n}$, respectively. As a limiting case, we allow \langle\rangle as the type of sentences, i.e. expressions forming sentences when combined with zero further expressions. We also add a category of cumulative types: whenever $t_{1}, t_{2}, \ldots$ are types, $\left[t_{1}, t_{2}, \ldots\right]$ is the cumulative type including all expressions belonging to any of $t_{1}, t_{2}, \ldots{ }^{20} \mathrm{We}$ write ' $\langle e *\rangle$ ' to abbreviate the infinite string ' $\langle e\rangle,\langle e, e\rangle,\langle e, e, e\rangle, \ldots$ ' and similarly for other types. So $[\langle e *\rangle]$ is the cumulative type including every (first-order) predicate, of whatever adicity. Since cumulative types are types, the recursive clause for functional types now also yields new functional types. There is, for example, the functional type $\langle e,[e,\langle e *\rangle]\rangle$ of expressions forming sentences when combined with a name as their first argument and an expression of type $[e,\langle e *\rangle]$ as their second argument. Since

20 For the meta-language of his limit language, Williamson adds only one cumulative type, namely a cumulative infinite limit type $\lambda$, that comprises exactly the expressions belonging to any finite type (cf. ibid). For my purposes, it is simpler to use only smaller cumulative types at finite orders. There should be no objection to this. If we can form a cumulative infinite limit type like $\lambda$ and use expressions of types derived from it, surely we can also form a cumulative type comprising, say, only names and first-order predicates, and use expressions of types derived from it. 
among the latter, there are both names and predicates, such expressions accept, in the same argument place, both names and predicates.

We use this system of types to describe, in the first step, an ordinary, non-cumulative second-order language $L_{2}$. It includes for each type $\tau$ among $e,\langle e *\rangle$ a countably infinite stock of constants and variables. We write the constants using lowercase letters from the beginning of the alphabet, augmented with subscripts as required, and marking their type by a superscript. We adopt the same convention for variables except for choosing letters from the end of the alphabet. $L_{2}$ also includes the identity-predicate ' $=\langle e, e\rangle$, the usual quantifier symbols, connectives, and parentheses. Type-superscripts may be omitted if there is no risk of ambiguity, and parentheses may be omitted or added according as readability is improved.

$L_{2}$ does not include any cumulative devices, so we may call it a pure second-order language. In all relevant ways, it is exactly the kind of language the higher-orderist needs to formulate his generalized semantics for the first-order language $L_{1}$. If we extend it by copies ' $\forall_{0} x^{e}$ ' and ' $\exists_{0} x^{e}$ ' of the quantifiers in $L_{1}$, we can formulate this version of $\left(\mathrm{GA}_{1}\right)$ :

$\left(\mathrm{GA}_{1}^{*}\right) \forall y^{e} \exists_{o} x^{e} x^{e}=y^{e}$

Given the requisite amount of syntax and set-theory, we can go on to formulate in $L_{2}$ a higher-orderist generalized semantics for $L_{1}$ that includes $\left(\mathrm{GA}_{1}{ }^{*}\right)$.

I shall now argue that independently of the admissibility of cumulative expressions, (DIST) should be rejected. The reason is that although the identity-predicate ' $=$ ' itself does not apply at the level of second- and higher-order quantification, higher-order analogues of that predicate do apply. Using these higher-analogues of the identity-predicate, we can construct higher-order counterparts of $\left(f_{1}\right)$ and thereby obtain higher-order analogues of $\left(\mathrm{MORE}_{1}\right)$. I maintain that these are similar enough to $\left(\mathrm{MORE}_{1}\right)$ that they should be taken to contradict the spirit of Absolutism.

We wish to extend $L_{2}$ by non-cumulative higher-order cousins of the identity-predicate connecting predicates and predicate variables of a given adicity. These expressions should then belong to the types $\langle\langle e\rangle,\langle e\rangle\rangle,\langle\langle e, e\rangle,\langle e, e\rangle\rangle$, etc. We shall officially write them '= $=\langle e\rangle,\langle e\rangle\rangle$, , $=\langle\langle e, e\rangle,\langle e, e\rangle\rangle$, etc., but often let context fix the type. What should we take these predicates to mean? For present purposes, we can think of them in either of three ways. Firstly, we can take them as primitive expressions subject to the inference rules analogous to the standard rules for identity. Where ' $a$ ' and ' $b$ ' belong 
to some type $\tau$ among $\langle e\rangle,\langle e, e\rangle, \ldots$ and $\Phi^{a / b}$ is the result of replacing zero or more occurrences of ' $b$ ' in $\Phi$ by ' $a$ ':

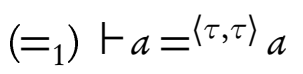

$\left(={ }_{2}\right) \vdash a=\langle\tau, \tau\rangle b \rightarrow\left(\Phi \rightarrow \Phi^{a / b}\right)$

Secondly, we can extend $L_{2}$ by third-order variables of type $\langle\langle e\rangle\rangle(\langle\langle e, e\rangle\rangle, \ldots)$ and quantifiers binding them. The expression ' $x{ }^{\langle e\rangle}=y^{\langle e\rangle}$ ' can then be taken to abbreviate the corresponding indiscernibility condition: $\forall z^{\langle\langle e\rangle\rangle}\left(z^{\langle\langle e\rangle\rangle}\left(x^{\langle e\rangle}\right) \leftrightarrow z^{\langle\langle e\rangle\rangle}\left(y^{\langle e\rangle}\right)\right)$, and similarly for the other types. Thirdly, since $L_{2}$ is an extensional language, coextensiveness implies indiscernibility, so we can take the predicates simply to abbreviate the relevant co-extensiveness condition, so that, for example, ' $x{ }^{\langle e\rangle}=y^{\langle e\rangle}$ ' abbreviates ' $\forall x^{e}\left(x^{\langle e\rangle}\left(x^{e}\right) \leftrightarrow y^{\langle e\rangle}\left(x^{e}\right)\right)$ '. Under both the second and third option, the rules $\left(={ }_{1}\right)$ and $(=2)$ are derivable.

Now suppose we include in $L_{2}$ a copy ' $\forall_{o} x\langle e\rangle$ ', of a monadic second-order quantifier of some second-order language. We may wonder whether from the perspective of $L_{2}$, that quantifier expresses an unrestricted, absolute form of monadic second-order generality. Roughly speaking, that is, we may wonder whether there is something in the range of $L_{2}$ 's monadic second-order quantifier that is distinct from anything in the range of ' $\forall{ }_{o} x^{\langle e\rangle}$. The informal talk of being distinct from anything in that range here can be regimented by means of ' $=\langle\langle e\rangle,\langle e\rangle\rangle$ ':

$\left.\left(\neq_{2}\right) \forall_{o} x^{\langle e\rangle} \ldots \not\langle e\rangle,\langle e\rangle\right\rangle x\langle e\rangle$

By putting a monadic second-order variable in the empty argument place and binding it with an existential quantifier, we obtain

$\left(\mathrm{MORE}_{2}\right) \exists y^{\langle e\rangle} \forall_{o} x^{\langle e\rangle} y^{\langle e\rangle} \neq\langle\langle e\rangle,\langle e\rangle\rangle x^{\langle e\rangle}$

It seems to me that it would be very implausible to discount this claim as irrelevant to the spirit of Absolutism on the grounds that it does not operate with the notion of distinctness as it occurs in $\left(\mathrm{GA}_{1}\right)$ and $\left(\mathrm{MORE}_{1}\right)$. It seems much more plausible to interpret this sentence as saying, in a sense that is relevant to the spirit of Absolutism, that the quantifier ' $\forall_{o} x^{\langle e\rangle}$ ' expresses only a restricted form of (monadic, second-order) generality. If so, then (DIST) should be rejected. Analogues of the ordinary identity- 
predicate allow for the formulation of analogues of $\left(\mathrm{MORE}_{1}\right)$ that contradict the spirit of Absolutism. ${ }^{21}$

We may thus turn to the second step in my argument. I shall argue that once we are allowed to use cumulative resources, we can formulate another variant on $\left(\neq_{1}\right)$, employing a cumulative higher-order counterpart of the identity-predicate. We can then use that expression to formulate a cumulative higher-order version of $\left(\mathrm{MORE}_{1}\right)$ as our regimentation of (MORE). Just as we took $\left(\mathrm{MORE}_{2}\right)$ to contradict the spirit of Absolutism, it seems to me, we should take that regimentation to do so as well. And since it turns out to be derivable in the semantics of the cumulative higher-orderist, the introduction of cumulative resources into higher-orderism is in this way seen to undermine the spirit of Absolutism.

We form the language $L_{2 \equiv}$ by extending $L_{2}$ with an identity-like symbol of type $\langle[e,\langle e *\rangle],[e,\langle e *\rangle]\rangle$, which we shall write ' $\equiv$ ' to make it easier to distinguish from the previous ones. In contrast to these, ' $\equiv$ ' is cumulative in both its argument places: it accepts both singular terms and predicates of any adicity as arguments.

What should we take ' $\equiv$ ' to mean? For present purposes, we may either take it as a new primitive expression, subject to certain inference rules, or we may take it as given an explicit definition. If we introduce ' $\equiv$ ' as a primitive, it should be governed by whatever rules render it as identity-like as possible, consistent with its non-standard syntax. It seems clear then, that it should satisfy at least the following two rules. Where ' $a$ ' and ' $b$ ' belong to $[e,\langle e *\rangle]$ :

$\left(\equiv_{1}\right) \vdash a \equiv a$

$\left(\equiv_{2}\right) \vdash a \equiv b \rightarrow\left(\Phi \rightarrow \Phi^{a / b}\right)$

(When ' $a$ ' and ' $b$ ' belong to different types, substituting one for the other sometimes produces ill-formed results. The rule $\left(\equiv_{2}\right)$ is therefore to be understood as restricted to well-formed instances. ${ }^{22}$ )

$21 \quad$ Could a higher-orderist dig their heels in and simply insist that $\left(\mathrm{MORE}_{2}\right)$ and its ilk are irrelevant to Absolutism? Although such a view would seem very unattractive, I know of no reason to think that it would have to somehow turn out to be internally incoherent. However, I think that Williamson could not happily resort to such a position, since he himself exploits the analogy between the identity-predicate and its higher-order counterparts in a way similar to how I have just used it; cf. e.g. Williamson, 2013, 263ff.

22 Absent contexts other than ' $\equiv$ ' in which expressions of different types can be exchanged without loss of grammaticality, our rules leave open the truth-value of any specific sentences in which ' $\equiv$ ' connects expressions of different types. We consider these in more detail in the next section. 
If we wish to instead introduce ' $\equiv$ ' by definition, we can extend $L_{2 \equiv}$ by variables of type $\langle[e,\langle e *\rangle]\rangle$ and allow these to be bound by quantifiers. We may then let ' $a \equiv b$ ' simply abbreviate the indiscernibility condition: $\forall z^{\langle[e,\langle e *)]\rangle}\left(z^{\langle[e,\langle e *)]\rangle}(a) \leftrightarrow\right.$ $\left.z^{\langle[e,\langle e *)]\rangle}(b)\right)$. Given a suitable comprehension scheme for the new quantifiers, the rules $\left(\equiv_{1}\right)$ and $\left(\equiv_{2}\right)$ are then derivable. ${ }^{23}$

We now use ' $\equiv$ ' to formulate a regimentation of (MORE). Let us include in $L_{2 \equiv}$ copies ' $\forall_{o} x^{e}$ ' and ' $\exists_{o} x^{e}$ ' of the first-order quantifiers of $L_{1}$. We can then formulate a cumulative variant on $\left(\neq_{1}\right)$ to express a condition of distinctness from anything in the range of $L_{1}$ 's quantifier:

$\left(\neq_{C}\right) \forall_{o} x^{e}-\not \equiv x^{e}$

Since ' $\equiv$ ' accepts expressions of type $\langle e\rangle$ as arguments, we can now put a monadic second-order variable in its empty argument place in $\left(\neq_{C}\right)$, and bind it with an existential quantifier. We then obtain the following regimentation of (MORE):

$\left(\mathrm{MORE}_{C}\right) \exists y^{\langle e\rangle} \forall_{o} x^{e} y^{\langle e\rangle} \not \equiv x^{e}$

Given $\left(\equiv_{1}\right)$ and $\left(\equiv_{2}\right)$, this sentence is derivable by broadly standard Russell-style reasoning in second-order logic. ${ }^{24}$ I maintain that like $\left(\mathrm{MORE}_{2}\right)$, it is similar enough to $\left(\mathrm{MORE}_{1}\right)$ that it should be taken to contradict the spirit of Absolutism.

23 Absent a meaningful notion of co-extensiveness defined for $[e,\langle e *\rangle]$ and thus names and predicates alike, unlike its non-cumulative cousins, ' $\equiv$ ' cannot be introduced as an abbreviation of a co-extensiveness condition.

24 Proof: We define a first-order predicate ' $r$ ' ${ }^{(e)}$ ' as follows, with ' $x^{e}$ ' ranging over everything: $r^{\langle e\rangle}\left(x^{e}\right) \leftrightarrow_{d f} \forall x^{\langle e\rangle}\left(x^{\langle e\rangle} \equiv x^{e} \rightarrow \neg x^{\langle e\rangle}\left(x^{e}\right)\right)$. Assume for reductio that for some ${ }_{0}$ object $r^{e}, r^{\langle e\rangle} \equiv r^{e}$. Now $r^{\langle e\rangle}\left(r^{e}\right) \vee \neg r^{\langle e\rangle}\left(r^{e}\right)$. Assume $r^{\langle e\rangle}\left(r^{e}\right)$. Then by definition $\forall x^{\langle e\rangle}\left(x^{\langle e\rangle} \equiv r^{e} \rightarrow \neg x^{\langle e\rangle}\left(r^{e}\right)\right)$, so in particular $r^{\langle e\rangle} \equiv r^{e} \rightarrow \neg r^{\langle e\rangle}\left(r^{e}\right)$. By assumption, $r^{\langle e\rangle} \equiv r^{e}$, so $\neg r^{\langle e\rangle}\left(r^{e}\right)$, contradicting our assumption of $r^{\langle e\rangle}\left(r^{e}\right)$. So $\neg r^{\langle e\rangle}\left(r^{e}\right)$. Then by definition, $\neg \forall x^{\langle e\rangle}\left(x^{\langle e\rangle} \equiv r^{e} \rightarrow \neg x^{\langle e\rangle}\left(r^{e}\right)\right)$. However, assume $x^{\langle e\rangle} \equiv r^{e}$. Since $r^{\langle e\rangle} \equiv r$, it follows by $\left(\equiv_{1}\right)$ and $\left(\equiv_{2}\right)$ that $x^{\langle e\rangle} \equiv r^{\langle e\rangle}$. Since $\neg r^{\langle e\rangle}\left(r^{e}\right)$, by another application of $\left(\equiv_{2}\right), \neg x^{\langle e\rangle}\left(r^{e}\right)$. So $x^{\langle e\rangle} \equiv r^{e} \rightarrow \neg x^{\langle e\rangle}\left(r^{e}\right)$. Since $x^{\langle e\rangle}$ was arbitrary, $\forall x^{\langle e\rangle}\left(x^{\langle e\rangle} \equiv r^{e} \rightarrow \neg x^{\langle e\rangle}\left(r^{e}\right)\right)$. But then by definition, $r^{\langle e\rangle}\left(r^{e}\right)$. Contradiction. So $r^{\langle e\rangle} \not \equiv r^{e}$, and since $r^{e}$ was arbitrary, $\forall_{o} x^{e} r^{\langle e\rangle} \not \equiv x^{e}$. $\left(\mathrm{MORE}_{C}\right)$ follows by existential generalization on $r^{\langle e\rangle}$. The proof assumes that the comprehension scheme for the second-order quantifiers of $L_{2 \equiv}$ allows impredicative instances including our new ' $\equiv$ '. Specifically, the legitimacy of the definition of ' $r\langle e\rangle$, and/or the subsequent existential generalization on it depend in effect on this impredicative instance of the comprehension schema: $\exists y^{\langle e\rangle} \forall x^{e}\left(y^{\langle e\rangle}\left(x^{e}\right) \leftrightarrow \forall z^{\langle e\rangle}\left(z^{\langle e\rangle} \equiv x^{e} \rightarrow \neg z^{\langle e\rangle}\left(x^{e}\right)\right)\right)$. Could the higher-orderist reject such impredicative instances of comprehension? I think not; such a move would appear to undermine the whole motivation for higher-orderism, since the initial Russell-style argument depends on an impredicative instance of (CPI Formal 1). 


\section{Cumulative identity}

The extent to which cumulative higher-orderism's commitment to $\left(\mathrm{MORE}_{C}\right)$ is a departure from the spirit of Absolutism depends on the strength of the analogy between ' $\equiv$ ' and '=', and thus $\left(\mathrm{MORE}_{C}\right)$ and $\left(\mathrm{MORE}_{1}\right)$. Given the unfamiliarity of cumulative devices in general and ' $\equiv$ ' in particular, it may not be very easy to get a clear sense of the strength of this analogy. This section therefore examines the behaviour of ' $\equiv$ ' and its relation to ' $=$ ' in more detail. I argue that there is no dissimilarity between ' $\equiv$ ' and '=' that could undermine the analogy between $\left(\mathrm{MORE}_{C}\right)$ and $\left(\mathrm{MORE}_{1}\right)$.

We note first that ' $\equiv$ ' shares the distinctive structural features of the identity relation. In particular, it expresses an equivalence relation in the sense that in addition to the reflexivity rule $\left(\equiv_{1}\right)$, symmetry and transitivity rules are derivable for ' $a$ ', ' $b$ ', ' $c$ ' in $[e,\langle e *\rangle]$ :

$\left(\equiv_{3}\right) \vdash a \equiv b \rightarrow b \equiv a$

$\left(\equiv_{4}\right) \vdash(a \equiv b \wedge b \equiv c) \rightarrow a \equiv c$

The proofs are exactly analogous to the corresponding proofs for '='.

Admittedly, the reflexivity, transitivity, and symmetry of the ordinary identityrelation can also be expressed in a non-schematic way by means of quantifications like $\forall x \quad x=x$ and $\forall x \forall y(x=y \rightarrow y=x)$. The structural features of ' $\equiv$ ' are not thus expressible in $L_{2 \equiv}$. The reason is that we do not have any variables that range over an entire cumulative type. So although we can express the reflexivity of $\equiv$ with respect to type $e$ by ' $\forall x^{e} x^{e} \equiv x^{e}$ ', and with respect to type $\langle e\rangle$ by ' $\forall x^{\langle e\rangle} x^{\langle e\rangle} \equiv x^{\langle e\rangle}$ ', and so on, we cannot express by a single sentence the reflexivity of $\equiv$ tout court.

We can remove even this disanalogy by moving to a new language $L_{2 C}$ extending $L_{2 \equiv}$ by what I shall call semantically cumulative variables. These are variables that belong to a cumulative type $\left[t_{1}, t_{2}, \ldots\right]$ without belonging to any of the accumulated types $t_{1}, t_{2}, \ldots$. Roughly speaking, they are intended to range over the entirety of values of the variables from the accumulated types. We may use underlined lowercase letters from the end of the alphabet for these variables, and express, for example, the reflexivity of $\equiv$ in $L_{2 C}$ as follows: ${ }^{25}$

Although the introduction of such variables could perhaps in principle be rejected by a cumulative
higher-orderist, it is important to see how natural their introduction is once syntactically cumu-
lative expressions have been introduced. For absent semantically cumulative variables, we have 
$\left(\equiv_{5}\right) \forall \underline{x}^{[e,\langle e *\rangle]} \underline{x}^{[e,\langle e *\rangle]} \equiv \underline{x}^{[e,\langle e *\rangle]}$

With respect to their structural features, then, ' $\equiv$ ' and '=' seem exactly analogous.

Moreover, whenever ' $\equiv$ ' connects two singular terms, the resulting sentence is true iff the corresponding sentence with ' $=$ ' is true. More generally, any given sentence in which ' $\equiv$ ' connects two expressions of the same non-cumulative type is true just in case the result of replacing ' $\equiv$ ' with a suitable non-cumulative identity-like predicate is true. That is, for $\tau$ among $e,\langle e *\rangle$ :

$\left(\equiv_{6}\right) \vdash a^{\tau} \equiv b^{\tau} \leftrightarrow a^{\tau}=\langle\tau, \tau\rangle b^{\tau}$

Let us then turn to the somewhat stranger contexts of ' $\equiv$ ' in which it connects expressions of different types; call them cross-type identifications. Our derivation of $\left(\mathrm{MORE}_{C}\right)$ has shown that in conjunction with the rest of the higher-orderist's logic, $\left(\equiv_{1}\right)$ and $\left(\equiv_{2}\right)$ already have substantive general implications concerning cross-type identifications: roughly speaking, at least one item in type $\langle e\rangle$ cannot be identified with any item in type $e$. The truth-values of all specific cross-type identifications, however, are left open by our theory. That is, where $\tau$ and $\sigma$ are different types among $e,\langle e *\rangle$, no sentence of the form $\left\ulcorner a^{\tau} \equiv b^{\sigma}\right\urcorner$ is either derivable or refutable from our rules. ${ }^{26}$ This is exactly parallel to the situation for the logic of ' $=$ ': where $a$ and $b$ are distinct singular terms, $\ulcorner a=b\urcorner$ is neither derivable nor refutable from the logical rules alone. Of course, if we are given some sentences $\ulcorner\Phi(a)\urcorner,\ulcorner\neg \Phi(b)\urcorner$ as premises, we can infer $\ulcorner a \neq b\urcorner$ from them. But in just the same way, for $a$ and $b$ in $[e,\langle e *\rangle]$ and given sentences $\ulcorner\Phi(a)\urcorner$, $\ulcorner\neg \Phi(b)\urcorner$ as premises, we may also infer $\ulcorner a \not \equiv b\urcorner$ from them. So far then, no relevant disanalogy between '=' and ' $\equiv$ ' has emerged.

cumulative predicates with application conditions defined for a range of items that cannot be swept out by a single variable. It seems more than odd to think that it should be impossible to add variables that can take values from the entire application range of the predicates. I shall henceforth assume that semantically cumulative variables are no more problematic than syntactically cumulative expressions.

26 It is clear that our rules do not permit the derivation of any theorem of the form $\ulcorner a \equiv b\urcorner$ where $a$ and $b$ are distinct expressions. A fortiori, they do not permit the derivation of any such theorem where $a$ and $b$ belong to different types. It may not be as obvious that $\left(\equiv_{2}\right)$ does not, as it stands, allow the derivation of any negation of a cross-type identification. But note that so far, ' $\equiv$ ' is the only cumulative predicate in our language. So any well-formed cross-type instance of $\left(\equiv_{2}\right)$ will be a formula like ' $a \equiv b \rightarrow(b \equiv c \rightarrow a \equiv c)$ ' where the substitution in the consequent occurs in the scope of ' $\equiv$ '. As a result, we could only obtain the negation of a cross-type identity from such a premise given another negation of a cross-type identity to start with. For essentially the same reason, we also cannot derive negations of cross-type identifications appealing to the proposed indiscernibility definition of ' $\equiv$ '. 
Perhaps one might be tempted to argue for such a disanalogy along the following lines. ${ }^{27}$ A cumulative counterpart of the identity-predicate like ' $\equiv$ ' is most naturally thought of on the model of the disjunction of the relevant non-cumulative identity-like predicates. Now when we think of ' $\equiv$ ' in this way, then it trivially produces a false sentence whenever it is fed expressions of different types as arguments. But that makes the crucial claims of distinctness from which $\left(\mathrm{MORE}_{C}\right)$ is inferred importantly dissimilar to the claims of distinctness from which $\left(\mathrm{MORE}_{1}\right)$ is inferred. For the latter claims of distinctness are not trivial in this way. And since $\left(\mathrm{MORE}_{C}\right)$ is now seen to be merely an immediate consequence of a perfectly trivial claim, it would be implausible to consider it as contradicting the spirit of Absolutism. For surely it would be disingenuous to interpret the spirit of Absolutism in such a way that it is straightforwardly inconsistent with a mere triviality.

My response is that the envisaged disjunctive understanding of ' $\equiv$ ' is incompatible with how I have introduced the expression, and that an understanding that is in line with how I have introduced ' $\equiv$ ' does not trivialize cross-type identifications in the way described. I suggested two alternative ways to introduce ' $\equiv$ '. The first is to take it as primitive, subject to the inference rules $\left(\equiv_{1}\right)$ and $\left(\equiv_{2}\right)$. These rules do not trivialize cross-type identifications, but tie them to predications involving the relevant terms. As a result, there are non-trivial considerations that can be brought to bear on the question of the truth or falsity of a given cross-type identification.

Consider first the question of the identity between objects $a$ and $b$. In order to decide the question, we may ask whether there is a predicate that, under a uniformation interpretation, is defined for both $a$ and $b$, and applies to $a$ but not to $b$. Since the cumulative higher-orderist allows cumulative predicates, a counterpart of that consideration applies to questions of cross-type identity. For instance, to decide whether $a^{e} \equiv b^{\langle e\rangle}$, we may ask whether there is a predicate of type $\langle[e,\langle e\rangle]\rangle$ - whose application conditions are accordingly defined for both $a^{e}$ and $b^{\langle e\rangle}$ - which, under a uniform interpretation, applies to $a^{e}$ but not to $b^{\langle e\rangle}$.

The second way of introducing ' $\equiv$ ' I offered is through an explicit definition in terms of the corresponding indiscernibility condition. This definition also does not trivialize cross-type identifications, but ties them to the existence of discriminating properties. To decide whether a given cross-type identification is true, we may ask whether there is a property of type $\langle[e,\langle e\rangle]\rangle$ - whose exemplification conditions accordingly are defined

$27 \quad$ Thanks here to [blinded]. 
for both $a^{e}$ and $b^{\langle e\rangle}$ - which $a^{e}$ has but $b^{\langle e\rangle}$ does not have. In the same way, the existence of discriminating properties bears on questions of first-order identity: we ask whether there is a property whose exemplification conditions are defined for both $a$ and $b$ and which $a$ has and $b$ does not have.

Finally, it is not obvious whether some cross-type identifications might not be true. ${ }^{28}$ Consider some way for a thing to be, say, wise, and the corresponding property, wisdom, conceived of as a bona fide object, i.e. something properly designated in a formal language by a singular term. It is not absurd to think that if $\equiv$ is to be the closest thing to identity that is defined for a range comprising both objects and ways for objects to be, then it should be the case that wisdom $\equiv$ is wise. Such a view might allow a cumulative higher-orderist to hold that most of the ways for things to be recognized in $L_{2 \equiv}$ are "identical" to things in the range of $L_{1}$ 's quantifier - the exception being the Russell-style ways for things to be. Indeed, a non-classical cumulative higher-orderist who endorses a naive comprehension schema for properties at the cost of some truths of classical logic could in this way argue for the negation of $\left(\mathrm{MORE}_{C}\right)$ :

$\left(\mathrm{GA}_{C}\right) \forall \underline{y}^{[e,\langle e *\rangle]} \exists_{o} x \underline{y}^{[e,\langle e *\rangle]} \equiv x$

Whatever the overall merits or demerits of such a view, it would thereby underwrite a stronger form of Absolutism than is consistent with a classical cumulative higherorderism such as Williamson's.

I conclude that ' $\equiv$ ' is not problematically dissimilar to ' $=$ ', and that the analogy between $\left(\mathrm{MORE}_{1}\right)$ and $\left(\mathrm{MORE}_{C}\right)$ is accordingly strong enough that $\left(\mathrm{MORE}_{C}\right)$ should be taken to contradict the spirit of Absolutism.

\section{Expressive Difficulties}

One of Williamson's arguments against Relativism is related to the peculiarity we noted in $\$ 2$ concerning the most natural ways an Absolutist might attempt to express their

28 It should be noted that the option of holding things of different types in general distinct is plausible at most for what we may call pure types, i.e. types that are neither cumulative, nor derived from cumulative types. If we allow semantically cumulative variables, we of course obtain some examples of true cross-type identifications. In particular, we should have the result that, for example $\forall x^{e} \exists \underline{y}^{[e,\langle e *)]} x^{e} \equiv \underline{y}^{[e,\langle e *)]}$. Moreover, for higher-level analogues of ' $\equiv$ ' which also apply to expressions of functional types derived from cumulative types, we shall also have true cross-type identifications where neither argument is of a cumulative type. For example, for the counterpart of 'E' in type $\langle[\langle e\rangle,\langle[e,\langle e\rangle]\rangle],[\langle e\rangle,\langle[e,\langle e\rangle]\rangle]\rangle$ we should have that $\forall x^{\langle e\rangle} \exists y^{\langle[e,\langle e\rangle]\rangle} x^{e} \equiv y^{\langle[e,\langle e\rangle]\rangle}$. 
view. Prima facie, the obvious way to do that is to utter a sentence like 'It is possible to quantify over absolutely everything'. Now if Absolutism is true, then we can interpret the Absolutists's use of the quantifier phrase 'absolutely everything' in the absolutist way they intend, and under such an interpretation, the Absolutist has said something relevant and true. But if Absolutism is false, then we cannot so interpret the utterance, and our Absolutist, far from making a relevant claim that is unfortunately true, has made a true but irrelevant claim. While that is perhaps a somewhat strange dialectical situation, it seems that at least by their own lights, the Absolutist can express their intended thesis.

Williamson suggests that the Relativist is in a considerably worse position (cf. 2003, \$5). The idea is this. Suppose the Relativist attempts to state his view by uttering the negation of the Absolutist's sentence, i.e. 'It is impossible to quantify over absolutely everything'. That sentence can express what the Relativist intends it to express only on the assumption that Relativism is false. And in that case, what the sentence expresses is also false, as one would have hoped. But if Relativism is true, the sentence simply says something unintended. Whatever restricted domain the quantifier 'absolutely everything' is interpreted as ranging over, the sentence then says that quantification restricted in that way is impossible. But that is not something the Relativist wants to proclaim to be impossible, and rightly so, for it evidently is not impossible. It seems that if the Absolutists cannot help but say something true, but at least say what they want if their view is right, the Relativists cannot help but say something false, and say what they wants only if their view is wrong.

Of course, even if successful, this argument does not show that standard Relativism is incoherent. It shows only that a natural first idea for stating the view is incoherent. Relativists might respond in two ways. They might simply resist the urge to produce a general claim supposed to capture their view, limiting themselves to claims like

$\left(\mathrm{MORE}_{1}\right) \exists y \forall_{o} x x \neq y$

describing individual languages like $L_{1}$ as expressing only a restricted form of generality. Although these claims seem to instantiate a common pattern, crying out for generalization, Relativists might simply reject any suggestion that there is a true generalization of which all these claims are mere special cases. ${ }^{29}$ Admittedly, while internally coherent, this kind of quietist positions may be less than fully satisfactory. Alternatively, Rela-

$29 \quad$ Button 2010 puts forth a view like this. 
tivists can try to devise some other way of formulating a general thesis that can capture their view without collapsing into incoherence. ${ }^{30}$

Whether or not Relativists can find a convincing solution to the problem, it appears that cumulative higher-orderists face exactly the same sort of difficulty. Their proposal requires that from any legitimate language, we can move to a meta-language that includes quantifiers of an order higher than any order of quantification found in the object-language. So like Relativists, they seem committed to a kind of inexhausibility thesis; only the higher-orderist's thesis concerns the entire hierarchy of orders of quantification. The most natural way to attempt to express the view is by uttering a sentence like: 'It is impossible to quantify over absolutely everything in the entire hierarchy of higher and higher orders of quantification.' Clearly, this sentence is no better off than the Relativist's doomed 'It is impossible to quantify over absolutely everything'.

Of course, this does not show that cumulative higher-orderism is incoherent. It shows only that a natural first idea for stating the relativist component of the view is incoherent. Like Relativists, higher-orderists might respond in two ways. They might simply resist the urge to produce a general claim supposed to capture the Relativist element of their view, limiting themselves to claims like

$\left(\operatorname{MORE}_{C}\right) \exists y^{\langle e\rangle} \forall_{o} x^{e} y^{\langle e\rangle} \not \equiv x^{e}$

describing individual languages like $L_{1}$ as expressing only a limited form of generality. Although these claims seem to instantiate a common pattern, crying out for generalization, higher-orderists might simply reject any suggestion that there is a true generalization of which all these claims are mere special cases. Again, the view seems to be internally coherent, but to the same extent as its Relativist cousin, it also seems less than fully satisfactory. Alternatively, higher-orderists can try to devise some other way of formulating a general thesis capturing their intended form of Relativism. It is not obvious how they might do so, but if they manage it, that would answer the objection just presented. However, it is to be expected (though I cannot prove) that if any such means were to be found, it would also give the standard Relativist a way of formulating their thesis without falling into incoherence.

I conclude that, as far as adequate formulation of their overall view is concerned, standard Relativists and cumulative higher-orderists are in a very similar situation.

$30 \quad$ See Fine 2006 for one proposal. 


\section{Interpretative Limitations}

Williamson also criticizes Relativism for painting an unattractive picture of parts of logical, metaphysical, and mathematical discourse (cf. Williamson, 2003, 415ff, 435). As I mentioned in the introduction to this paper, such discourse appears to provide us with numerous examples of utterances intended as absolutely general. As a result, the interpretations of such utterances that the Relativist can offer seem, from a pre-theoretic standpoint, quite weird and implausible. In this section, I investigate whether the interpretations offered by the cumulative higher-orderist should be considered similarly weird and implausible.

Let me first try and bring out as clearly as I can that and why the Relativist's interpretations seem weird. ${ }^{31}$ Consider any of the following sentences:

(6) Everything is self-identical.

(7) If something $x$ is identical to something $y$, then this is necessarily so.

(8) Everything is necessarily identical to something.

(9) Everything is either abstract or concrete.

From the point of view of a Relativist's meta-language and semantics, utterances of any of (6)-(9) can be interpreted only as restricted to some less than all-inclusive domain $D$. In typical cases, any such interpretation seems to simply be a misinterpretation. Consider the formalization

(10) $\forall x(A x \vee C x)$

of (9) in a formal first-order language $L_{1}$. Assume we have formulated a Relativist semantics in a first-order meta-language $L_{2}$, which we may suppose include the predicates 'is abstract' and 'is concrete' with their usual meanings. Now consider the following two sentences of $L_{2}$

(11) $\forall x(x$ is concrete $\vee x$ is abstract $)$

$31 \quad$ Although I myself agree that they do seem weird, I have encountered some resistance to this view in discussion. So it may be worth stressing two points. Firstly, it is important to guard against a misunderstanding. As I see it, the issue concerns the plausibility of certain interpretations from a pre-theoretic standpoint. Among other things, this means that 'interpretation' here must not be understood in any theoretically loaded sense like assignment of semantic values, or some such, but is to be taken in an intuitive, pre-theoretic sense. Secondly, for the purposes of my argument, it does not matter much whether the following considerations do show that the Relativist's interpretations should seem implausible. For my claim will simply be that if they do, then similar considerations establish an analogous, if less drastic verdict on the cumulative higher-orderist's interpretations. 
(12) $\forall x \in D\left(x\right.$ is concrete $_{D} \vee x$ is $\left.\operatorname{abstract}_{D}\right)$

where $D$ is the domain with respect to which we are interpreting $L_{1}$, and 'abstract $D$ ' and 'concrete ${ }_{D}$ ' mean the same as their subscript-free counterparts except in that their application conditions are defined only over $D$. Evidently, the generalization expressed by (12), as well as every generalization expressed by a version of (12) in which some other set is referred to in place of $D$, bears an interesting relationship to the generalization expressed by (11). Roughly speaking, (12) is obtained from (11) through replacing the quantifier ' $\forall x$ ' by a proper restriction of it, and replacing the predicates by counterparts with accordingly restricted application conditions. I shall say the generalization expressed by (12) is a mere restriction of that expressed by (11), and conversely that the latter is a mere expansion of the former.

The important point is that the claim expressed by (12), because it is a mere restriction of that expressed by (11), seems a strange target for metaphysical inquiry, and relatedly a strange claim to put forth as the upshot of a metaphysical investigation. The claim expressed by (11) seems a much more interesting and natural claim to focus on. At least at a first, and again, perhaps somewhat naive glance, it therefore seems implausible to interpret a metaphysician using the $L_{1}$-sentence (10) to put forth (part of) his metaphysical theory as having endorsed the claim expressed by (12), not (11). Of course, if we try to specify, in a meta-language for our meta-language, an intended interpretation of (11), it, too, will turn out to express a mere restriction of a yet more encompassing counterpart. In this way, saying that everything is abstract or concrete becomes something of a metaphysisyphean task.

To illustrate the point another way, imagine a necessitist like Williamson uttering (8). In effect, the Relativist would interpret him as saying that everything except perhaps some things outside $D$ is necessarily (identical to) something. This seems very strange. From a naive point of view, one expects the Relativist to then ask our necessitist what his view is with respect to the things outside $D$. The kind of conversation that would then unfold seems not worth having. N: 'I think that everything, in- or outside $D$, is necessarily something.' R: 'Oh, I see, you think that everything in your domain of discourse $D^{\prime}$ is necessarily something. But what about the things outside $D^{\prime}$ ?' $\mathrm{N}$ : 'Yes, they too. Indeed, everything, and so in particular everything outside $D^{\prime}$, is necessarily something.' R: 'I see, so everything in $D^{\prime \prime} \ldots$ '

Thankfully, disputes in logic and metaphysics rarely take this shape. But it is not obvious why interpreting speakers in the Relativist's way should not invite this kind 
of tiring sequence of questions and responses. Relatedly, it is not obvious why the Relativist's interpretations of the pertinent utterances are not cases of strange misinterpretation. So at first glance, it seems that Relativism paints a somewhat disconcerting picture of significant parts of logico-metaphysical inquiry.

However, it seems to me that the same kind of problem also arises for the cumulative higher-orderist, although only in a somewhat less dramatic form. Specifically, for some of universal generalizations of $L_{1}$, it seems that from the perspective of the cumulative meta-language $L_{2 C}$, they express mere restrictions of more encompassing claims. This is so in particular for $L_{1}$-generalizations involving only logical vocabulary.

Consider the $L_{1}$-sentence

(13) $\forall x x=x$

On the cumulative higher-orderist's semantics given in $L_{2 C}$, it expresses the claim that (14) $\forall x^{e} x^{e}=x^{e}$

Now, as we have seen, using cumulative resources, we can express a version of the notion of identity that is defined for both objects and ways for objects to be. That notion $\equiv$ seems to relate the ordinary notion of identity in much the same way as the notion of abstractness relates to that expressed by 'abstract ${ }_{D}$ ' above. The ordinary identity-predicate ' $=$ ' means the same as ' $\equiv$ ', except in that its application conditions are defined only for objects.

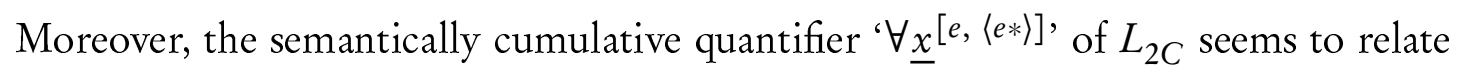
to ' $\forall x^{e}$ ' as expansion to restriction in much the same as ' $\forall x$ ' above does to ' $\forall x \in D$ '. Indeed, from the perspective of $L_{2 C}$, we could also say that (13) expresses the claim that $^{32}$

(15) $\forall \underline{x}(\mathrm{OBJ}(\underline{x}) \rightarrow \underline{x} \equiv \mathrm{OBJ} \underline{x})$

where 'OBJ $(\underline{x})$ ' is defined by ' $\exists y^{e} \underline{x} \equiv y^{\prime}$ and thus applies to all and only objects, and ' $\underline{x} \equiv_{\mathrm{OBJ}} y$ ' is defined by 'OBJ $(\underline{x}) \wedge \mathrm{OBJ}(y) \wedge \underline{x} \equiv \underline{y}$ ' and thus applies to all and only identical objects.

In this way, we can formulate mere expansions for any given universal generalization in $L_{1}$ as long as it contains only first-order predicates that have natural higherorder analogues. Clearly, this is not the case for every generalization. For example,

32 For readability, I have here dropped the type-superscript $[e,\langle e *\rangle]$ from the underlined, cumulative variables. 
it does not seem as though the abstract/concrete distinction has any obvious counterparts at higher orders of quantification. Nevertheless, it holds for quite a few interesting and contentious logico-metaphysical theses, such as the necessity of identity and existence. More cases show up when we consider a second-order language as objectlanguage. They include, for example, the claim that nothing has contradictory properties $\left(\forall x^{e} \neg \exists x^{\langle e\rangle}\left(x^{\langle e\rangle}\left(x^{e}\right) \wedge \neg x^{\langle e\rangle}\left(x^{e}\right)\right)\right)$, or the conclusion of the Russell Paradox $\neg \exists z^{\langle e, e\rangle} \forall x^{\langle e\rangle} \exists x^{e} \forall y^{e}\left(x^{\langle e\rangle}\left(x^{e}\right) \leftrightarrow z^{\langle e, e\rangle}\left(x^{e}, y^{e}\right)\right)$.

It would be an exaggeration to claim in conclusion that with respect to the difficulty of interpreting apparently absolutely general discourse in logic, mathematics, or metaphysics, a cumulative higher-orderist is in as worrisome a position as the Relativist. The cases in which they may be thought to interpret speakers in an implausibly restricted fashion are less wide-spread than those arising for Relativism, and it is not obvious that the restricted interpretations are as implausible-looking as those given by the Relativist. Nevertheless, the difference appears to be one of degree, and a smaller one than one might have expected.

\section{Conclusions}

The attempt to develop a generalized semantic theory in a first-order language for an object-language that expresses absolute generality runs into a version of Russell's Paradox. Standard Relativism concludes that absolute generality is impossible. Williamson 2003 criticizes that view on a number of grounds, including that it is by its own lights not properly expressible, and that it yields an unsatisfactory picture of parts of logical, metaphysical, and mathematical inquiry. To avoid Relativism, Williamson appeals to a hierarchy of higher and higher orders of quantification, which, in his recent book, he extends to transfinite and cumulative orders of quantification. I have argued that the emerging view has implications strongly reminiscent of the distinctive commitments of standard Relativism and thus gives up on much of the spirit of Absolutism. Moreover, versions of the two criticisms of Relativism just mentioned also apply on the cumulative higher-order view.

Cumulative higher-orderism is not thereby shown to be wrong, of course. (Indeed, I incline to think it is correct.) What I hope to have shown, however, is that it does not let us be as much of a Generality Absolutist as we might have thought and hoped. 
Even according to cumulative higher-orderism, there is not just everything. There is everything, and then some.

\section{References}

George Boolos. To be is to be a value of a variable (or to be some values of some variables). The Journal of Philosophy, 81(8):430-449, 1984.

George Boolos. Nominalist platonism. Philosophical Review, 94:327-344, 1985.

Tim Button. Dadaism: Restrictivism as militant quietism. Proceedings of the Aristotelian Society, 110:387-398, 2010.

Wolfgang Degen and Jan Johannsen. Cumulative higher-order logic as a foundation for set theory. Mathematical Logic Quarterly, 46:147-170, 2000.

Kit Fine. Relatively unrestricted quantification. In Agustín Rayo and Gabriel Uzquiano, editors, Absolute Generality, pages 20-44. Oxford University Press, Oxford, 2006.

Salvatore Florio. Unrestricted quantification. Philosophy Compass, 9/7:441-454, 2014. Øystein Linnebo. Sets, properties, and unrestricted quantification. In Agustín Rayo and Gabriel Uzquiano, editors, Absolute Generality, pages 149-178. Oxford University Press, Oxford, 2006.

Øystein Linnebo and Agustín Rayo. Hierarchies ontological and ideological. Mind, 121:269-308, 2012.

Arthur Prior. Objects of Thought. Clarendon Press, Oxford, 1971.

Agustín Rayo. Beyond plurals. In Agustín Rayo and Gabriel Uzquiano, editors, Absolute Generality, pages 220-254. Oxford University Press, Oxford, 2006.

Agustín Rayo and Gabriel Uzquiano. Toward a theory of second-order consequence. Notre Dame Journal of Formal Logic, 40(3):1-11, 1999. 
Agustín Rayo and Gabriel Uzquiano. Introduction. In Agustín Rayo and Gabriel Uzquiano, editors, Absolute Generality, pages 1-20. Oxford University Press, Oxford, 2006.

Agustín Rayo and Stephen Yablo. Nominalism through de-nominalization. Nôus, 35(1):74-92, 2001.

Timothy Williamson. Everything. Philosophical Perspectives, 17:415-465, 2003.

Timothy Williamson. Modal Logic as Metaphysics. Oxford University Press, Oxford, 2013. 